\title{
Bildverfremdung gegen Bildverehrung. Zu einigen Darstellungsstrategien in der nördlichen Tafelmalerei des Spätmittelalters
}

\author{
LieselotTe E. SAURMA-JELTSCH
}

\section{Einführendes}

Im Rahmen des Themas »Auffiihrung « und >Schrift«« sich mit bildender Kunst des Mittelalters zu beschäftigen, scheint zunächst eine Auseinandersetzung nahezulegen mit der Beziehung dieser Kunst zum Theater, etwa zum geistlichen Schauspiel. Tatsächlich bestehen ja eine Reihe von Verwandtschaften zwischen den beiden Medien: Ihr gemeinsames Ziel ist die Übermittlung von theoretischen, didaktischen und unterhaltsamen Inhalten in visualisierter Form. ${ }^{1}$ Aber nicht allein in ihrer Vermittlungsaufgabe, sondern auch in den Inhalten und selbst in ihren Anliegen entsprechen sie sich. Die Passionsgeschichte und Heiligenviten sind hier wie dort die zentralen Themen, die sich meist aus denselben oder zumindest ähnlichen Quellen speisen. ${ }^{2}$ Die Forschung, die sich in der Kunstgeschichte in eigenartigen Wellenbewegungen entweder für eine besonders enge Verbindung zwischen den beiden Medien ausspricht ${ }^{3}$ oder im Gegenteil eine solche bagatellisiert ${ }^{4}$, hat

1 Zur allgemeinen Bibliographie vgl. Götz Pochat, Theater und bildende Kunst, Graz 1990. S. 36 f., Anm. 129.

2 Zur Gemeinsamkeit der Strukturen vgl. Frank O. Büttner, Imitatio Pietatis. Motive der christlichen Ikonographie als Modelle der Verähnlichung, Berlin 1983, bes, in der Einleitung; Sixten Ringbom, Icon to Narrative, 2. Aufl. Doornspijk 1984, bes. S. 11-23; Pochat (Anm. 1), S. 51; Marie-Leopoldine Lievens-de Waegh, »Le langage de l'image chez les sprimitifs flamands . Parallèles avec le langage verbal «, Revue des archéologues et historiens d'art de Louvain 20 (1987), S. 153-177.

3 Besonders betont von Emile Mâle, L'Art religieux du XII siècle en France, Paris 1922. Kapitel IV und V.

4 Dazu bes. Pochat (Anm. 1), S. 37 ff. - Zur Schwierigkeit einer direkten Übertragbarkeit von Begriffen und Mustern vgl. Robert Suckale, "Süddeutsche szenische Tafelbilder um 1420-1450. Erzählung im Spannungsfeld zwischen Kult- und Andachtsbild«, in: Wolfgang Harms (Hrsg.), Text und Bild. Bild und Text. DFG-Symposion 1988, Germanistische Symposien-Berichtsbände 11, Stuttgart 1990, S. 15-34, bes. S. 15; vgl. dazu auch Martin Stevens, »The Intertextuality of Late Medieval Art and Drama «, New Literary History 22 (1991), S. 317-337: Die Verbindung versteht Stevens als eine grundsätzlich mißverständliche, da die Umsetzung in das jeweilige Medium eine Bedeutungs- und Inhaltsverschiebung bewirke; vgl. bes. Memlings Turiner Passionsbild, das in der kunsthistorischen Literatur als Paradebeispiel für eine Parallele zum Drama mit seiner Kumulierung von szenischen Bühnen gilt; dazu Pochat (Anm. 1), S. 41 ff. 
mindestens im Motivischen, in der Verwendung bestimmter Requisiten, aber auch in den erzählerischen Strukturen ${ }^{5}$ unmittelbare Beziehungen feststellen können.

Nicht die Frage der Relation von Schauspiel und bildender Kunst wird jedoch im folgenden thematisiert, sondern es sollen bestimmte Präsentationslösungen in der religiösen nordeuropäischen Tafelmalerei besprochen werden, die ein gleichsam theatralisiertes oder >aufgeführtes< Bild ergeben. Der in den betreffenden Darstellungen zu beobachtende Verzicht auf die Wiedergabe eines mimetischen, der geschauten Wirklichkeit entsprechenden Eindrucks wird nicht als Unvermögen der Maler verstanden, sondern als ein bewußt verfremdendes Formmittel interpretiert. So kommt eine Distanz zwischen Betrachter und Bild zustande, durch die das Bild - wie auf einer Bühne - auf einen ihm eigenen Wirklichkeitsausschnitt begrenzt wird.

Die Intentionen für den Aufbau einer solchen Distanz zwischen Betrachter und Bildinhalt dürften vielfältig sein; ${ }^{6}$ im Zusammenhang der vorgegebenen Fragestellung möchte ich nur eine verfolgen, nämlich die Veränderung der Rezeptionsanweisung. Da es sich ja ausschließlich um religiöse Bilder handelt, soll mit solchen Mitteln der Verfremdung die Identifikation des Betrachters mit dem Bild verhindert werden: Er soll nicht in das Bild >eintreten «, sondern durch dieses auf eine andere, projektive Ebene geführt werden. Das Bild dient lediglich als Einstieg; es verweist auf die Beschäftigung mit dem Thema, das als gleichsam von der Tafel zu ihm sprechend präsentiert wird.

Mit diesem Verweis auf einen >eigentlichen` Inhalt wird nun auf eine ältere Bildauffassung rekurriert. Die innerbildliche Realität im Sinne eines eigenen Wirklichkeitsausschnitts wird zurückgenommen, um als oberste Instanz die Heilige Schrift bewahren zu können. Dadurch wird die im ganzen Mittelalter gültige Stellung des biblischen Wortes als eine dem Bild übergeordnete Autorität zurückgewonnen.

Im folgenden soll der Prozeß dieser mit ganz unterschiedlichen Mitteln vorgenommenen Verfremdung und der damit verbundenen Zuwendung zur theatralischen Repräsentation dargestellt werden. Als Beispiel dient die Untersuchung der Veränderungen, welche ein von Jan van Eyck entwickeltes Thema bei seinen Nachfolgern erfährt. Zunächst werden die Mittel der Verfremdung analysiert werden müssen; das heißt, es wird zu fragen sein, in welcher Weise der Bildgegenstand den Betrachter einbezieht oder sich von ihm distanziert. Daraufhin wird zu untersuchen sein, wie weit der Verzicht auf die Wiedergabe der geschau-

5 Hans Belting, Das Bild und sein Publikum im Mittelalter, Berlin 1981, bes. S. 77 ff., mit weiterführender Literatur. Zur Beziehung zwischen >tableau vivant $<$ und bildender Kunst vgl. Pochat (Anm. 1), S. 83 f.

6 Dazu etwa Erwin Panofsky, Early Netherlandish Painting, 2 Bde.. Cambridge/Mass. 1953, bes. Bd. I, S. 309 ff. - Einen Eindruck von der Komplexität des Themas, bei dem zweifellos auch wirtschaftliche und soziologische Komponenten eine Rolle spielen, vermittelt Craig Harbison, »Realism and Symbolism in Early Flemish Painting «, Art Bulletin 46 (1984), S. 588-602, bes. S. 589 f.; ders., Jan van Eyck. The Play of Realism, London 1991 , bes. S. $198 \mathrm{ff}$. 
ten Wirklichkeit mit meiner These des saufgeführten< Bildes einer Erklärung zugeführt werden kann. Dabei wird es gelten, Veränderungen der Bildfunktionen zu beachten und den Verweischarakter im Sinne eines Einstiegs in die Beschäftigung mit übergeordneten Themen zu prüfen.

\section{Die Entwicklung zum >autonomen< Bild}

Aktuell werden kann unsere Fragestellung erst mit dem Prozeß der allmählichen Entwicklung einer bildnerischen Autonomie, mit dem wir uns daher kurz beschäftigen müssen. Gregors des Großen berühmtes Diktum über die Funktion der Bilder als Hilfsmittel für die illitterati bezeichnete ja nicht so sehr einen mehr oder weniger eingeschränkten Benutzerkreis - waren die illitterati meist doch kaum in der Lage, komplexere Bilder zu >lesen $<$ - als daß es genau jenen Gebrauch von Bildern umschrieb, der noch während des ganzen Mittelalters weitgehend seine Gültigkeit behielt. ${ }^{7}$ Vornehmste Aufgabe des Bildes war, wie auch Gregor dies forderte, Lehre und Erinnerung der Heiligen Schrift zu sein, was zu einer besonderen und sehr engen Bindung an das Wort Gottes führte. Dieses blieb als oberste Instanz bewußt und verschaffte den Bildern ihre eigene $>$ Heiligkeit «, die - wie das tiefe Unverständnis zeigt, mit dem die Karolinger auf die byzantinische Bilderfrage reagierten ${ }^{8}$ - einen falschen Bildergebrauch im Sinne einer Idolisierung ausschloß.

Die Intensivierung der Diskussion im 12. und 13. Jahrhundert ${ }^{9}$ belegt, daß man sich der Gefahr dennoch und wohl erneut bewußt war, wie sehr Bilder zu Mißverständnissen führen, ja sogar die Verwechslung des Abbildes mit dem Urbild provozieren können. Vorwürfe der Götzenanbetung, der Statuenliebe ${ }^{10}$ und der Verblendung durch Bilder häufen sich nicht umsonst genau in jener Zeit, in der die Bilder eine neue Realitätskraft gewinnen, nämlich in der Frühgotik. ${ }^{11}$

Der Wandel zu dem neuen Bildverständnis zog sich über mehrere Jahrhunderte hin. Ein wichtiger Schritt ist dabei in der zunehmenden Emanzipation des Erzählens, insbesondere in Andachtsbildern, zu sehen, war doch damit eine verstärkte Ansprache an den Betrachter verbunden. An dessen emotionale und sub-

7 Lawrence G. Duggan, »Was Art Really the Book of the Illiterate? «, Word and Image 5 (1989), S. 227-251; Celia M. Chazelle, »Pictures, Books and the Illiterate. Pope Gregory I's Letters to Serenus of Marseilles«, Word and Image 6 (1990), S.138-153.

8 Vgl.dazu Lieselotte E. Saurma-Jeltsch, »Zur karolingischen Haltung gegenüber dern Bil derstreit «, in: 794 - Karl in Frankfurt. Ausstellungskatalog zur 1200-Jahrfeier, Sigmaringen 1994, S. 69-72; immer noch überzeugend: Gert Haendler, Epochen karolingischer Theologie. Eine Untersuchung üher die karolingischen Gutachten zum bvaantinischen Bilderstreit, Theologische Arbeiten 10, Berlin 1958, bes. S. $74 \mathrm{ff}$.

9 Michael Camille, The Gothic Idol. Ideology and Image-Making in Medieval Art. Cambridge/Mass. 1989, bes. S. $197 \mathrm{ff}$.

10 Berthold Hinz, „Statuenliebe. Antiker Skandal und mittelalterliches Trauma«, Marbur ger Jahrbuch für Kunstgeschichte 22 (1989), S. 135-142, bes. S. 139 ff.

11 Camille (Anm. 9), S. 203 ff. 
jektive Kräfte wird mit den Bildern appelliert. Im Sinne einer imitatio bieten sie eine affektive, ja sogar körperliche Übernahme des Bildgegenstandes an, wodurch zwar die Distanz des Betrachters zum Inhalt des Bildes in einem projektiven Vorgang aufgehoben wird, aber keineswegs diejenige zum Bild selbst: Es bleibt in einer klar identifizierbaren, eigenen Bildräumlichkeit. ${ }^{12}$

Mit der Forderung nach einer getreuen Nachahmung der Natur, wie sie als erster Leon Battista Alberti in seinem Traktat >Della pittura (1435) auch in der Theorie formulierte ${ }^{13}$, hat sich allerdings der Gegenstand der Malerei im Vergleich zum mittelalterlichen Bild grundsätzlich geändert. Es ist - so Alberti - zu einem Fenster geworden, hat den Durchblick geöffnet in eine Welt, welche der natürlichen Wahrnehmung des Betrachters entspricht. Christopher Braider ${ }^{14}$ sieht in diesem Wandel eine Ablösung von zwei Wahrheitsbegriffen, nämlich demjenigen, der sich einzig durch die Heilige Schrift definierte und demjenigen, der sich allein auf die sinnliche Wahrnehmung stützte: $\gg[\ldots]$ what is this window if not the art of painting itself as distinguished from the textual sources to which it owes its topics and authority? «. ${ }^{15} \mathrm{Ja}$, er zielt auf einen noch viel radikaleren Paradigmenwechsel mit der Behauptung, Malerei porträtiere nicht nur, sondern sie sei das, was sie darstelle. Dies würde bedeuten, daß das Bild selbst zu einer Wahrheit wird, die vorher allein dem Wort Gottes zukommen konnte, eine These, auf die noch ausführlicher einzugehen sein wird.

In der dem Konzept Albertis entsprechenden Bildgestaltung der Frührenaissance werden zur Nachahmung der Natur die neuen Erfahrungen mit der Optik eingesetzt. Dadurch, daß die Physiologie des Auges den Horizont des für die Menschen Ersichtlichen bestimmt, wird dieses Organ auch zum grundlegenden Maßstab jeglicher Erkenntnis. Die gemalten Objekte werden durch ihre perspektivische Wiedergabe in eine objektiv überprüfbare Ordnung eingefügt. Zwischen dem Objekt und dem Subjekt des Betrachters oder Malers besteht infolgedessen ein klar definiertes Verhältnis. Beispielsweise wird bei der LuccaMadonna des Jan van Eyck ${ }^{16}$ (Abb. I) der Betrachter zum Bezugspunkt; er ist es, der die räumliche Ordnung des Bildes definiert. >Der Mensch als Maß aller Dinge ${ }^{17}$ prägt folglich die Erfahrung der Objektwelt in ihrer Objektivität, zu-

12 Vgl. dazu Hans Belting, Bild und Kult. Eine Geschichte des Bildes vor dem Zeitalter der Kunst, München 1990, S. 459 ff.; David Freedberg, The Power of Images. Studies in the History and Theory of Response, Chicago u. London 1991, bes. S. $161 \mathrm{ff}$.

13 Zur Übergangsstellung Albertis vgl. Christopher Braider, Refiguring the Real. Picture and Modernity in Word and Image, 1400-1700. Princeton/N. J. 1992, bes. S. 20-36: Michael Baxandall, Giotto and the Orators. Oxford 1971. S. 121 ff.; Hans Belting.Ikone und Bilderzählung in der venezianischen Malerei. Frankfurt/M. 1985, S. 31 ff.; ders. (Anm. 12), S. $524 \mathrm{f}$.

14 Braider (Anm. 13), S. 69

15 Ebd.

16 Frankfurt, Städel: Inv. Nr. 944; vgl. dazu Jochen Sander, Niederländische Gemälde im Städel. 1400-1550, Kataloge der Gemälde im Städelschen Kunstinstitut Frankfurt am Main 2, Mainz 1993, S. 245-263, mit Literatur.

17 Peter Burke, Die Renaissance in Italien. Sozialgeschichte einer Kultur zwischen Tradition und Eifindung, Berlin 1984, bes. S. $194 \mathrm{ff}$. 
gleich allerdings wird er nun in seiner Betrachtung auf einen bestimmten StandPunkt festgelegt - man denke etwa an eine der ältesten perspektivisch richtigen Darstellungen, das Trinitätsfresko von Masaccio in Sta. Maria Novella in Florenz: > Richtig< präsentiert sich das Wandbild nur zu bestimmten Tageszeiten und in einem genau definierten Abstand. ${ }^{18}$

Die Erfahrbarkeit der Bildwelt ist somit mit Erfahrbarkeit der Welt des Betrachters kongruent. ${ }^{19}$ In einem ganz besonderen Maße wird diese Übereinstimmung in den Werken der frühen Niederländer nachvollziehbar, in denen die perspektivische Wiedergabe des Raumes verbunden wird mit einer perfekten sinnlichen Beschreibung des optischen und taktilen Eindrucks der Objekte. ${ }^{20}$ In der Lucca-Madonna werden dem Betrachter das Fenster mit den Butzenscheiben, die Nische mit dem im Licht glänzenden messingenen Lavabo, die mit Flüssigkeit gefüllte Glaskaraffe, in der sich ein Fenster spiegelt, wie auch der mit Brokatstoff verkleidete Thron mit den Löwenlehnen in einer solchen Deutlichkeit präsentiert, als ob er sie greifbar vor sich hätte. Diese Illusion wird überdies durch die Konstruktion des Bildraumes noch weiter gesteigert, in den der Betrachter mit einbezogen ist: Das Gewölbe der Decke, die seitlichen Wände, aber auch der Teppichboden sind so beschnitten, daß sie sich im Betrachterraum fortzusetzen scheinen. Dank dieser Konstruktion erfährt er den Blick auf die Madonna und den Raum, als ob er an dessen Eingang stünde und dazugehörte. Bedenken wir nun, daß dieses Bild folglich eine Begegnung face-à-face mit dem Heiligen - mit der Madonna und dem Kind - gestattet, die sich überdies in einem scheinbar der Welt des Betrachters entsprechenden Raum abspielt, so wird erst die Kühnheit der Darstellung bewußt. Der Betrachter ist zum Mitakteur geworden, ja - so Jochen Sander - »die Bildaussage erschließt und vervollständigt sich erst mit der und durch die Anwesenheit des andächtigen Betrachters «.21 Das Bild wird infolgedessen erst dann saktiviert ، oder komplettiert, wenn der andächtige Betrachter in den Bildraum eintritt.

Ein solches Einbeziehen des Betrachters birgt nun genau jene Gefahr in sich. welche Christopher Braider für bereits überwunden und nichtig erklärt, nämlich die Identifizierung des Bildes mit der Wahrheit. Für die Lucca-Madonna würde dies nichts anderes heißen, als daß der Betrachter in einen Raum eintreten kann. in welchem er die Madonna mit Kind nicht als Bild, sondern in Realpräsenz sieht. Daß tatsächlich solche Verwechslungen vorgekommen sind und vor allem deren Gefährlichkeit den Zeitgenossen sehr bewußt war, ließe sich mit der - für ein an der mittelalterlichen Tradition geschultes Auge - Mißverständlichkeit der neuen Bildkonzeptionen erklären.

18 Martin Kemp, "Science, Non-Science and Nonsense. The Interpretation of Brunelleschi's Perspective«, Art History 1 (1978). S. 134-161.

19 Max Imdahl, »Überlegungen zur Identität des Bildes«, in: Odo Marquard u. Karlheinz Stierle (Hrsg.), Identität, Poetik und Hermeneutik 7, München 1979, S. 187--211, bes S. 191-195.

$20 \mathrm{Vgl}$. Harbison, Jan van Eyck (Anm. 6), S. $13 \mathrm{ff}$.

21 Sander (Anm. 16), S. 257. 


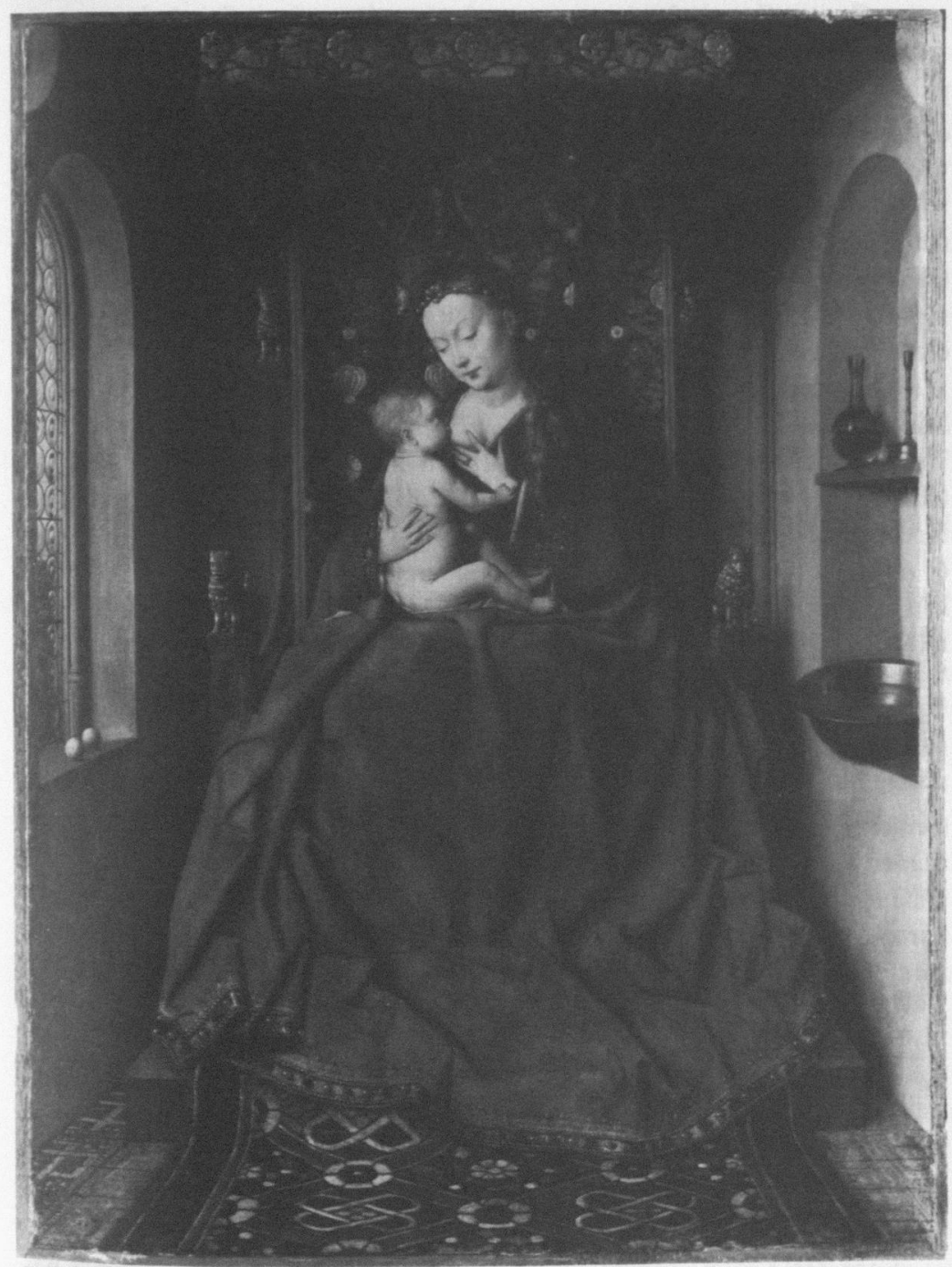

Abb. I: Jan van Eyck, Lucca-Madonna,

Städelsches Kunstinstitut, Frankfurt/M., Inv. Nr. 944

(Foto: Frankfurt, Ursula Edelmann). 


\section{Die Gefahr des >falschen` Bildes}

Der Vorwurf an die Malerei, daß sie eine Realität fingiere, die - bleibt sie doch eine gemalte - keine ist, und da $\mathrm{B}$ sie das, was nicht ist, zum Sein bringen könne, war ihr während des ganzen Mittelalters präsent. Bereits in der Antike war immer wieder vor ihrem falschen Schein gewarnt worden. ${ }^{22}$ Die Frührenaissance erst nahm dieses Paradoxon positiv auf - so lobt etwa Cennini, Giotto sei ein solches Genie gewesen, daß er Gegenstände wie die Natur wiedergegeben habe, ja sie sogar noch ähnlicher gemacht habe, so daß arme Leute gemeint hätten, es seien die Gegenstände selbst. ${ }^{23}$

Kein anderer Meister seiner Generation verstand die Verführungskunst der Malerei besser zu nutzen als Jan van Eyck. ${ }^{24}$ Die funkelnden Juwelen in seiner Tafel veranlaßten angeblich Diebe dazu, sich ihrer bemächtigen zu wollen. Kein anderer aber war sich zugleich der Gefahr des Verismus so sehr bewußt. Sein Streben galt denn auch nicht dem Abmalen der geschauten Wirklichkeit, sondern er versuchte, den Widerschein des Unsichtbaren im Sichtbaren wiederzugeben. ${ }^{25}$ Ein vergleichbares Verständnis formulierte etwa Nikolaus von Kues, der ebenfalls im Sichtbaren das Unsichtbare, das Unendliche und Göttliche sieht:

O Gott [...], daß ich sehe, Dein an Nichts gebundenes, nur in sich stehendes Antlitz sei das natürliche Angesicht jeder Naturart und -beschaffenheit, sei das Angesicht, welches die unbedingte und unbeschränkte Seinsheit jeden Seins, die Könnens- und Wissensumfassung alles Wißbaren ist! ${ }^{26}$

Gerade an solchen Vorstellungen scheint sich Jan van Eyck zu orientieren, der immer wieder den Verweischarakter gemalter , Wirklichkeiten ‘ heraushebt. Beinahe spöttisch setzt er für den intellektuellen Betrachter das oben erwähnte Paradoxon der Malerei ein und weist immer wieder darauf hin, daß das, was sie zum Sein zu bringen vermag, nicht wirklich ist. ${ }^{27}$ Das Spiel mit Realitäten ge-

22 Gute Zusammenfassung bei David Summers, Michelangelo and the Language of Art. Princeton/N.J. 1981, S. 45 ff.

23 Ebd., S. 50; Rudolf Preimersberger, "Zu Jan van Eycks Diptychon der Sammlung Thyssen-Bornemisza«, Zeitschrift für Kunstgeschichte 54 (1991), S. 459-489, bes. S. 466.

24 Harbison, Jan van Eyck (Anm. 6), bes. S. 158 ff. u. S. 198 ff.; Panofsky (Anm. 6), Bd. I, S. 137-139; Lotte Brand Philip, The Ghent Altarpiece and the Art of Jan van Eyck. Princeton 1971 , S. 163 f.u. S. 193 ff.

25 Harbison, Jan van Eyck (Anm. 6), S. 199, erklärt die unterschiedlichen Realismen in van Eycks Bildern mit dem Zusammenspiel von Auftraggeber/Gebrauchssituation und dem Vorgehen des Künstlers; als Lösung vermutet er, van Eyck habe Bilder mit mehrfacher Bedeutung hergestellt, die für ein variables Publikum gedacht waren.

26 Nikolaus von Kues, De visione Dei, Kapitel 7, zitiert nach: Von Gottes Sehen, hrsg. Elisabeth Bohnenstaedt, Schriften des Nikolaus von Cues, hrsg. Ernst Hoffmann [u.a.] im Auftr. d. Heidelberger Akademie der Wissenschaften, Philosophische Bibliothek. Leipzig 1947, Heft IV, S. 73 - - Zur Beziehung der niederländischen Kunst und Nikolaus von Kues vgl. Götz Pochat, Geschichte der Ästhetik und Kunsttheorie. Von der Antike bis zum 19. Jahrhundert, Köln 1986, S. 216-222; dagegen der pragmatische Ansatz von Harbison, Jan van Eyck (Anm. 6), S. 199 ff.

27 Preimersberger (Anm. 23), bes. S. 466 ff. u.S. 480 f.; Summers (Anm. 22), S. 50 ff. 
winnt gerade bei ihm einen übergeordneten, metaphysischen Bezug. Der Betrachter wird immer wieder an die Sätze des Nikolaus von Kues erinnert, verweisen doch die vielen von Jan van Eyck eingesetzten Spiegelbilder in ihren den Gesetzen der Optik folgenden Reflexen darauf, daß sie bloße Erscheinungen des an anderem Ort befindlichen Urbildes sein sollen. ${ }^{28}$ Wird damit nicht jene Erkenntnis des Cusanus verbildlicht, daß sich >im Spiegel der Ewigkeit ‘ das Abbild gleichsam als Schatten des Urbildes des Betrachters zeige?

Wenn einer in diesen Spiegel schaut, erblickt er seine Gestalt [...]. Und er meint, die Gestalt, die er in diesem Spiegel erblickt, sei die Abbildung seiner eigenen Gestalt [...]. Es ist zwar das Gegenteil davon der Fall. Was er in diesem Spiegel der Ewigkeit sieht, ist nicht Abbildung, sondern die Wahrheit, von der er, der Sehende, Abbild ist. ${ }^{29}$

Denn alles Abbild sei in der Wahrheit das Urbild, und Nikolaus vergleicht den Menschen mit einem lebendigen Schatten, zu dem Gott die Wirklichkeit darstelle. ${ }^{30}$

In den sich im schwarzen Stein des Hintergrunds spiegelnden Statuetten des Diptychons der Sammlung Thyssen-Bornemisza dürfte nicht nur, wie Rudolf Preimersberger gezeigt hat, »die körperliche Wirklichkeit [der Statuetten] für den Betrachter sich spiegelnd bewähren, [und] zugleich [...] der Prüfstein der [...] spektakulären Kunstleistung « ihres Malers sich befinden ${ }^{31}$, sondern ebenso sind sie - im Sinne des Cusanus - Belege für die Existenz des Urbildes, das im Abbild immanent vorhanden ist. In mehrfachen Brechungen erhält hier das Bild einen Wahrheitsanspruch, der aber nicht so sehr darin besteht, zu belegen, daß die Malerei selbst nun die Wahrheit sei, wie Braider dies behauptet, sondern im Gegenteil auf einen ParallelschluB verweist, wie er ja in der Argumentationsweise des Nikolaus von Kues üblich ist.

In den van Eyckschen Darstellungen geht es also nicht um das Abbilden einer autonomen Wirklichkeit, sondern um die Darstellung der in dieser Wirklichkeit sich spiegelnden Abbilder des Urbildes. Realitätsbrüche ${ }^{32}$ und Verweise auf austauschbare Realitäten sollen den Spiegelcharakter seiner Darstellungen erkennen lassen. Seinen Nachfolgern aber scheint diese sehr intellektuelle, artistische Verfremdung nicht genügt zu haben, kehren sie doch - wie wir noch sehen werden - zu den älteren Bildtypen zuruick.

Nicht zuletzt die Bilderkritik der Hussiten hatte die Gefahr vor >Götzenbildern erneut ins BewuBtsein gerufen. ${ }^{33}$ Im 15. Jahrhundert werden denn die Ermahnungen, Bilder nie zur Abgötterei, sondern lediglich als Instrument der Verehrung zu

28 Preimersberger (Anm. 23), S. 476.

29 Nikolaus von Kues, De visione Dei (Anm. 26), Kapitel 15, S. 102.

30 Zur Verfremdung des Urbildes im Spiegel vgl. Karl-Josef Pazzini, »Von Meister Eckharts , Bildung « zu Brunelleschis >Abbildung « «, in: Christian Rittelmeyer (Hrsg.). Bild und Bil dung, Wiesbaden 1991, S. 187-214, bes. S. 200.

31 Preimersberger (Anm. 23), S. 489.

32 Hans Belting u. Dagmar Eichenberger, Jan van Eyck als Eraähler, Worms 1983, bes. S. $46 \mathrm{ff} \mathrm{u.S.} 151 \mathrm{ff}$.

33 Horst Bredekamp, Kunst als Medium sozialer Konflikte. Bilderkämpfe von der Spätantike bis zur Hussitenrevolution, Frankfurt/M. 1975, S. 288 f.; Jacek Debicki, »Ein Beitrag zur Bildertheologie der vorhussitischen und hussitischen Zeiten in Böhmen «, Umení 40 (1992). S. 415-422. 
verwenden, immer zahlreicher und eindringlicher. ${ }^{34}$ Alle diese vehement gegen die Identifizierung des Abbildes mit dem Urbild gerichteten Aussagen beruhen auf der alten, eingangs erwähnten Auseinandersetzung der Kirche mit dem Bildergebrauch und dem Bilderverbot. Verschärft hat sich diese Problematik im Spätmittelalter noch durch die zunehmende private und von Laien gepflegte Frömmigkeit, die unter den Reformbewegungen im 15.Jahrhundert ein großes Ausmaß annahm. Über die >biedere`Suche nach der Veranschaulichung Gottes und iber den Bildermißbrauch schrieb schon Meister Eckhart: [...] aber etliche liute wellent got mit den ougen ansehen, alse sie ein rint ansehent.$^{35}$ Gott sei eben nicht mit den äußeren, sondern mit den inneren Augen zu sehen. Das von Menschen hergestellte Bild soll - so die Bildtheorie der Mystiker wie auch der späteren Reformbewegungen, etwa in der Art der devotio moderna - lediglich dem Einstieg in die Andacht dienen..$^{36}$

\section{Das Bild als Erfahrungsakt}

Reformbewegungen wie die devotio moderna ${ }^{37}$ betonen den neuen Wert einer Frömmigkeitserfahrung, die mit Hilfe der Visualisierung gewonnen werden kann. Entsprechende Äußerungen finden sich sowohl in der Predigtliteratur als auch in Anweisungen zum Gebrauch von Bildern. Auf deren Bedeutung für die Veranschaulichung von bestimmten Heilswahrheiten wird nicht selten sogar in den Predigten selbst hingewiesen. ${ }^{38}$ Geiler von Kaisersberg etwa rät dem From-

34 Michael Baxandall, Die Kunst der Bildschnitzer. Tilman Riemenschneider. Veit Stoß und ihre Zeitgenossen, München 1984, S. 63 f.; S. 64 ein Zitat aus dem anonymen Traktat Der Spiegel des Sünders, Augsburg 1475: Man solle bildnuß, nit für sich selbs, sunder von der wegen, der bildnuß sy seind [...] eren. Wann taetest du anders, als das du anbettest die bildnu $\beta$ christi und der heiligen $[\ldots]$ du begiengest die sunde der abgoeterei. Meister Eckhart: Deutsche Predigten, Nr. 14, zitiert nach Pazzini (Anm. 30), S. 188. Büttner (Anm. 2), S. 2; Pochat (Anm. 26), S. 201; Jean Wirth, "La naissance de Jesus dans le cœur: Etude iconographique «, in: La dévotion moderne dans les pays bourguignons et rhênans des origines à la fin du XVI siècle, Publications du centre européen d'études bourguignonnes (XIVe-XVIe s.) 29, Neuchâtel 1989, S. 149-158; Fritz O. Schuppisser, "Schauen mit den Augen des Herzens. Zur Methodik der spätmittelalterlichen Passionsmeditation, besonders in der Devotio Moderna und bei den Augustinem «, in: Walter Haug u. Burghart Wachinger (Hrsg.), Die Passion Christi in Literatur und Kunst des Spätmittelalters, Tüibingen 1993, S. 163-210.

37 Generell zur Beziehung zwischen bildender Kunst und devotio moderna vgl. Albert Chấtelet, "Hugo van der Goes et la dévotion moderne«, in: La dévotion moderne (Anm. 36). S. 129-139; Jean Rivière, "Robert Campin et son influence sur la devotio moderna à Bruxelles à la fin du XVe siècle«, ebd., S. 139-146; Lloyd Benjamin, „Disguised Symbolism Exposed and the History of Early Netherlandish Painting «. Studies in Iconography 2 (1976), S. 11-24.

38 Walter Dress, Die Theologie Gersons. Eine Untersuchung zur Verbindung von Nominalismus und Mystik im Spätmittelalter, Gütersloh 1931, bes. S. 116-119; vgl, etwa auch die Übergänge zum Schauspiel in der dramatischen Visualisierung von Predigern; dazu Michael Baxandall, Die Wirklichkeit der Bilder. Malerei und Erfahrung im Italien des 15.Jahrhunderts, 2. Aufl., Frankfurt/M. 1980, S. 64 f. 
men, anhand eines Bildes beispielsweise das Geheimnis der visitatio nachzuvollziehen: Das Bild solle betrachtet werden; und

[...] gedenck daran wie sie frölich gewesen seind [...] und erken das im glauben (recognoscere per fidem), darnach wan du es erkant hast, so hab ein gefallen daran $[\ldots]$ Zu dem dritten darnach so erzög dich gegen inen in üßerlicher eer erbeitung. Küß die bild $[\ldots]$ neig dich vor irem bild oder knüw darfür nider und rüff sie an $\left[\ldots . .{ }^{39}\right.$

Diese durch das Bild provozierten Formen emotionaler Anteilnahme entsprechen jenen Visualisierungsstufen, auf denen der Gläubige - gemäß gewissen Gebetsanleitungen - in unterschiedlichen Gefühlslagen jeweils verharren soll. ${ }^{40}$ Solche Affektzustände zu erreichen und damit nicht bloß ein physisch-psychisch begründetes Memorieren der Ereignisse zu fördern, sondern auf der höheren Erfahrungs- und Erlebensstufe auch die Nachahmung der Mysterien zu ermöglichen, ist Grundlage sowohl der Liturgie, des Schauspiels als auch der Bilder.

Wie Anweisungen zur meditativen Praxis wirken beispielsweise die zwei Versionen des heiligen Hieronymus in der Wüste, die uns von Gerard David überliefert sind. Die Frankfurter Darstellung ${ }^{41}$ (Abb. II) zeigt den Heiligen in einer Felslandschaft kniend vor einem gemalten Kreuzigungsbild, das an einem Baum hängt und unter dem sich der Löwe räkelt. Obwohl am Boden als Zeichen seiner Buße das abgelegte Kardinalsgewand liegt, scheint eine aktive Buße in diesem Bild zu fehlen. Dafür spricht offenbar auch die eigenartig abwehrende Haltung der linken Hand, was die bisherige Literatur zur Deutung veranlaßt hat, es handele sich bei dieser Darstellung um ein >domestiziertes Routinebild..$^{42}$ Die noch unversehrte Brust des Heiligen sowie die abwehrende Haltung der Linken, die den Gestus Mariens in der Verkündigung aufnimmt, sprechen nun allerdings dafür, daß es sich im Gegenteil um die Thematisierung einer bestimmten Form der Meditation und Auseinandersetzung handelt. Hier wird genau jenes Zwiegespräch festgehalten, das der Betrachter des Bildes ebenfalls führen sollte, stellt es doch den Heiligen in einem Zustande der conturbatio, einem emotional erwünschten Zustand der Verwirrung, dar, wie ihn Maria als erste Reaktion auf die Ansprache des Engels gezeigt hatte. ${ }^{43}$ In der wohl dem David-Umkreis zuzuschreibenden Hieronymus-Tafel der National Gallery in London ${ }^{44}$ (Abb. III) ist nun der Heilige in einer anderen Gefühlslage geschildert, hat er doch hier offen-

39 Das Evangelienbuoch mit uszlegung des hochgelerten Doctor Keiserspergs, StraBburg o.J., zitiert nach: L. Dacheux, Un réformateur catholique à la fin du XVe siècle. Jean Geiler de Keysersberg. Prédicateur à la cathédrale de Strasbourg 1478-1510, Straßburg 1876, S. 272.

40 Baxandall (Anm. 38), S. $61 \mathrm{ff}$

41 Frankfurt, Städel: Inv. Nr. 1091: dazu Sander (Anm. 16), S. 223-232; dort ältere Literatur.

42 John D. Farmer, "Gerard David's Lamentation and an anonymous St. Jerome «. Museum Studies 8 (1976), S. 51 f.; vgl. auch Sander (Anm. 16), S. 231 . Anm. 42.

43 Dazu Baxandall (Anm. 38), S. $66 \mathrm{ff}$.

44 London, National Gallery: No. 2596; Max J. Friedländer, Early Netherlandish Painting, Bd. VI/2, Leiden 1971, S. 109. Taf. 226: vgl. auch Sander (Anm. 16), S. 225. Anm. 15 dort weitere Literatur. 


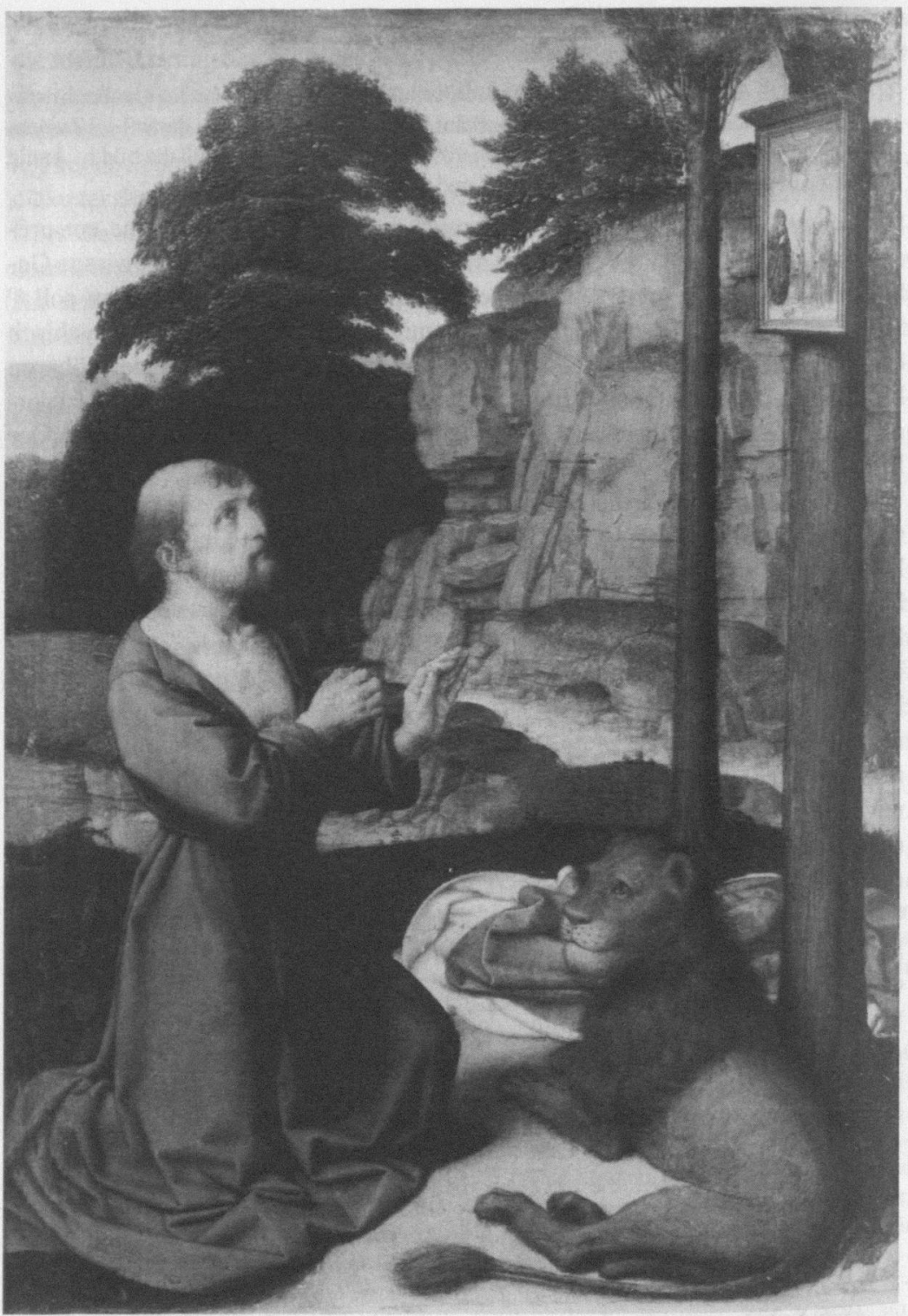

Abb. II: Gerard David, der Heilige Hieronymus in der Wüste, Städelsches Kunstinstitut, Frankfurt/M., Inv. Nr. 1091

(Foto: Frankfurt, Ursula Edelmann). 


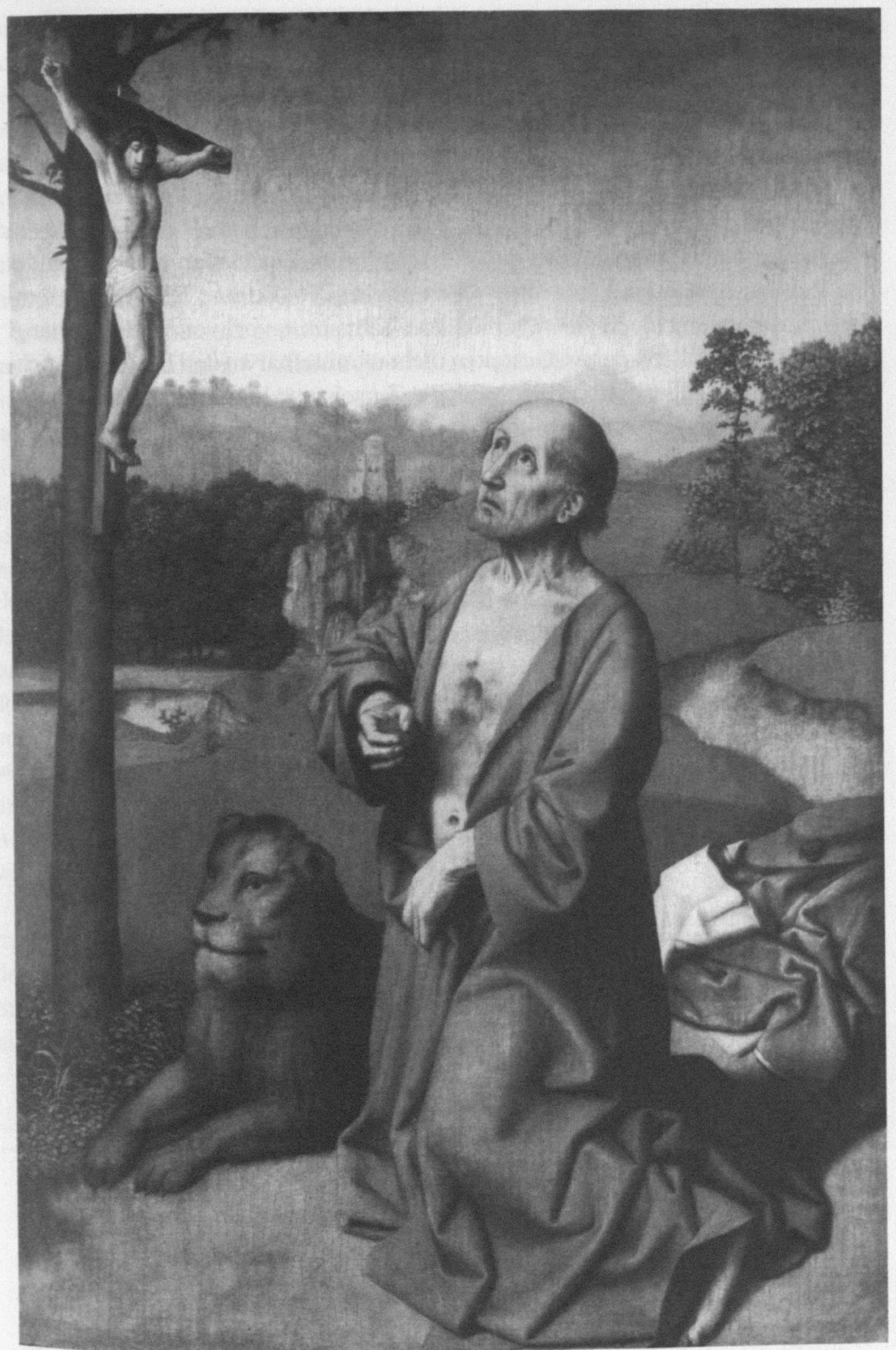

Abb. III: Umkreis Gerard David, Heiliger Hieronymus in der Wüste, London National Gallery, No. 2596

(Foto: London National Gallery). 
bar die Vision akzeptiert und sich ihr, wie die Wundmale und der Stein in seiner Hand zeigen, bereits unterworfen. In den beiden Tafeln ist Hieronymus infolgedessen in zwei unterschiedlichen Meditationsstufen gezeigt: Erst in der Phase des aktiven Mitleidens, der imitatio Christi, befindet er sich in jener Erkenntnisform, die es ihm ermöglicht - wie der am Baum aufscheinende Kruzifixus im Gegensatz zur gemalten Tafel des anderen Bildes deutlich macht - die Vision des Gekreuzigten zu sehen. ${ }^{45}$

In beiden Darstellungen wird der Betrachter zum Zuschauer, dem die Bilder eine Lektion im Umgang mit ihnen übermitteln: Die Akteure leben ihm exemplarisch die unterschiedlichen Formen von Teilnahme vor, von Verähnlichung. Obwohl sich die Hieronymus-Gestalten nicht unmittelbar an den Betrachter richten, sprechen sie dennoch zu ihm durch die Zurschaustellung ihrer nachahmenswerten Zustände. In ihrer Vorbildlichkeit dienen sie eher der Anleitung, als daß sie zur Identifikation auffordern, was sie an ein traditionelles Bildverständnis anknüpfen läßt.

Erst im Vergleich zu diesen Hieronymustafeln ist die ganz andere Funktion eines Bildes, wie sie Jan van Eyck mit der Lucca-Madonna (Abb. I) anstrebt, zu ermessen. Während bei der Lucca-Madonna der Betrachter durch sein eigenes Auge sich im Abbild selbst findet - ist doch sein Augenpunkt identisch mit dem Zentrum des Mysteriums, dem auf dem Schoß der Mutter sitzenden Kind -, wird er in den Hieronymustafeln auf Distanz gehalten. Die Bilder präsentieren sich ihm hier mit der Aufforderung zur Nachahmung. Es ist streng darauf geachtet, $\mathrm{da} ß$ die innere, visionäre Welt von der äußeren des Betrachters abgegrenzt wird. Nun wird wiederum - wie in der überkommenen Bildtradition - zu ihm gesprochen, ${ }^{46}$ er ist $\mathrm{Zu}$-Schauer und wird als solcher zur aktiven Teilnahme animiert.

\section{Der Weg von der Einsicht zur Ansicht}

Das Beispiel der Lucca-Madonna diente dazu, eine Bildauffassung bewußt $\mathrm{zu}$ machen, die zumindest bis in die Phase der sogenannten >konkreten $\triangleleft$ Kunst unseres Jahrhunderts gültig bleiben sollte, die sich zugleich aber radikal von allen älteren Konzepten absetzte. Wie sehr sich van Eyck dieser Ablösung bewußt war und deren Gefahr, falsche Wahrheiten zu produzieren, erkannt hatte, haben wir gesehen. Während nun der Betrachter bei Jan van Eyck virtueller Mitspieler in

45 Dagegen steht allerdings die Interpretation von Harbison, der in der Tafelmalerei der ersten Generation der Niederländer gerade eine Form von entmaterialisierter, daher der Vision zugänglicher Kunst sieht; vgl. Craig Harbison, "Visions and Meditations in Early Flemish Painting«, Simiolus 15 (1985), S. 87-118, bes. S. 117 f. - Dieser Deutung widerspricht die abwehrende Haltung des Heiligen in der Frankfurter Tafel, die auf keinen Fall als Zustand der Gottesschau interpretiert werden kann, wird doch diese erst in der absoluten Ruhe und Versenkung dem Auserwählten geschenkt.

46 Zum sprechenden Bild vgl. Belting (Anm. 5), bes. S. 126 ff.; vgl. auch ders. (Anm. 12), S. $459 \mathrm{ff}$. 
den Bildern bleibt und - wie spätere Vergleiche noch weiter verdeutlichen werden - zur Vermeidung einer Idolisierung andere Mittel angewandt werden, ist seine Rolle bei allen Nachfolgern unmißverständlich als diejenige eines $\mathrm{Zu}$ schauers festgelegt. Diese Rückkehr zu einem alten Bildverständnis soll zunächst an drei Tafeln aufgezeigt werden, welche das Thema der Lucca-Madonna aufgreifen. In unserem Zusammenhang ist vor allem zu prüfen, welche Bedeutung solche Veränderungen für den Gebrauch der Bilder haben, und ob dadurch tatsächlich, wie eingangs behauptet wurde, im Sinne einer Verfremdung eine Distanzierung zwischen Betrachter- und Bildwelt geschaffen werden soll.

In der nicht selten als genuines Werk Jan van Eycks angesehenen, sogenannten Ince-Hall-Madonna ${ }^{47}$ (Abb. IV) wird der rote Mantel Mariens zu einem bestimmenden Bildelement. Während nun allerdings Jan van Eyck dieses Motiv zur Distanzierung des Gnadenbilds vom Rest des Bildes benutzt, indem er mit Hilfe des Mantels und dessen scharfer Konturierung der heiligen Gruppe eine ungreifbare und überdimensionierte Monumentalität verleiht, ${ }^{48}$ wird es in der Ince-Hall-Madonna in ganz anderer Weise eingesetzt. Der Mantel in seiner mächtigen Breite, seiner unklaren Tiefenräumlichkeit, die zugleich die körperliche Gestalt Mariens in einer ungreifbaren Farbenflut versinken läßt, dient hier dazu, das gesamte Bild aus der räumlichen Erfahrungswelt des Betrachters herauszuheben, ihm eine eigene Gesetzlichkeit zu verleihen. Damit stimmt auch überein, daß nun der Blick zum Beobachter hin geöffnet ist, die Mutter das Kind präsentiert und dieses noch zudem das Buch der Bücher vorweist. Verzichtet wird somit auf die Einbeziehung des Betrachters als Akteur: Das Bild ist in seiner eigenen Räumlichkeit von derjenigen des Betrachters abgesetzt, es ist zur An-Schauung konzipiert mit dem eindeutigen Hinweis, daß hier repräsentiert wird.

Wesentlich effektiver wird die Distanzierung des Bildinhaltes aus der Betrachterwelt in einer anderen Version desselben Themas angeboten, nämlich in dem für Abt Wolfhard Strauss gemalten Madonnenbild (Abb. V).$^{49}$ In dieser in St. Emmeram in Regensburg aufbewahrten Tafel sind aus der Lucca-Madonna die folgenden Motive übernommen worden: die nährende Mutter mit dem Kind,

47 Melboume, National Gallery of Victoria: Felton Bequest, 1922. Vgl. dazu Stephan Lochner Meister zu Köln. Herkunft - Werke - Wirkung. Ausstellung, Köln 1993. Katalog 17, S. 262 f.; Ursula Hoff u. Martin Davies, The National Gallery of Victoria Melbourne. Brüssel 1971, Nr. 132, S. 29-50, dort ältere Literatur; hier, wie auch im Lochner-Kata$\log$, wird die Tafel als zeitgenössische Kopie eines Werkes von Jan van Eyck eingeordnet. Dagegen nimmt Sander (Anm. 16). S. 261, an, es handle sich um ein »Pasticcio, das in erheblichem Umfang gerade bei der Lucca-Madonna Anleihen macht «; ebd.. Anm. 68 weitere Beispiele der Nachfolge.

48 Auf die verschlüsselte Sakralität des Bildes macht besonders Sander (Anm. 16), S. 259 f.. aufmerksam.

$49 \mathrm{Vgl}$. dazu Alfred Stange, Deutsche Malerei der Gotik. Bd.X, Reprint Nendeln 1969, S. 102: Ernst Buchner, "Zur spätgotischen Malerei Regensburgs und Salzburgs «, Sitzungsherichte der Bayerischen Akademie der Wissenschaften. Phil.-hist. Klasse, München 1959, S. 3-9: Robert Suckale, »Das Znaimer Retabel. Zur künstlerischen Herkunft des Bildschnitzers". Osterreichische Zeitschrift für Kunst- und Denkmalpflege 42 (1988), S. 2-14, bes. S. 6 f. 
die Gewandbildung des marianischen Mantels, die Geschlossenheit der Gruppe sowie der mit Brokat überzogene Vorhang. Mit großer Nachdrücklichkeit jedoch wird darauf verwiesen, daß dieses Bild keine Darstellung einer Gnadenvision sein will. Mächtig scheint die Mutter Gottes vor ihrem Thron zu schweben; die winzige Gestalt des Stifters vor dem linken Bildrand steht gleichsam stellvertretend für den Betrachter, den er hin zu dem Gnadenbild weist. Die über dem Haupt der Mutter Gottes eine Krone haltenden Engel konkretisieren die Darstellung nicht allein als eine der Maria Regina, sondern zeigen auch, wie unerreichbar für den Betrachter diese Vision ist, nehmen sie doch das Thema einer revelatio auf und siedeln damit das gesamte Bild in den Himmelssphären an.

In sehr sublimer Weise schließlich wandelt Petrus Christus in seiner Frankfurter Madonna mit Kind und den Heiligen Hieronymus und Franziskus ${ }^{50}$ (Abb. VI) das van Eycksche Thema ab. ${ }^{51}$ Die Ausweitung des Darstellung im Sinne einer italienischen sacra conversazione auf eine von zwei Heiligen flankierte Madonna mit Kind ${ }^{52}$ hat der Szene einen feierlichen und repräsentativen Charakter verliehen. Im Gegensatz zu den beiden anderen Versionen - der Ince-Hall-Madonna wie auch der Strauss'schen - wird zwar auch hier, wie bei Jan van Eyck, mit den Mitteln der Zentralperspektive gearbeitet, wobei allerdings diese nun für eine ganz andere Aussage eingesetzt wird ${ }^{53}$ Hier wird nämlich dem Betrachter der Blick in einen nachvollziehbaren, der Größe der Mariengestalt entsprechenden Raum geöffnet, der jedoch gekennzeichnet ist als ein für ihn nicht betretbarer. Abgesetzt vom vorderen Bildrand erhebt sich der Thron, in dessen untere Stufe überdies als Beleg, daß es sich hier um ein Artefakt handelt, in Steinschrift die Künstlersignatur eingemeißelt ist. Die beiden Heiligen übernehmen die Rolle, Stellvertreter des Betrachters zu sein. Der Blick in den Himmel und in die kleinteilig gemalte Landschaft hinter dem Franziskus scheint die Irrealität dieser Darstellung eher zu verstärken als ihr eine eigene bildliche Autonomie zu verschaffen. Die bildparallele Anordnung, die Übergröße der Figuren und deren feierliche Haltung schaffen den Eindruck eines zum Bild erstarrten tableau vivant.

Jochen Sander ist zuzustimmen, wenn er den Vergleich zwischen der LuccaMadonna und derjenigen von Petrus Christus folgendermaßen resümiert: »Wo Jan van Eyck auf die Aufhebung von Bild- und Betrachterraum zielt, hält Petrus

50 Frankfurt, Städel: Inv. Nr. 920; Maryan W. Ainsworth (Hrsg.), Petrus Christus. Renaissance Master of Bruges, New York 1994, Nr.13, S. 136-141 [Katalog der Ausstellung im Metropolitan Museum of Art]; Sander (Anm. 16), S. 155-173, dort ältere Literatur.

51 Literatur zur negativen Bewertung dieser Abhängigkeit in Sander (Anm. 16), S. 162. Anm. 31 u. 32. - Allgemein zu dieser Beziehung vgl. Joel M. Upton, Petrus Christus. His Place in Fifteenth-Century Flemish Painting, Philadelphia 1990, S. 41, Anm. 50.

52 Zum Typus der sacra conversazione vgl. Sander (Anm. 16), S. 162, Anm. 35 u. 36; zur Inhaltsverschiebung vgl. Barbara G. Lane, The Altar and the Altarpiece, New York 1984. S. $23 \mathrm{f}$.

53 Zur Analyse dieser Unterschiede vgl. auch Upton (Anm. 51), S. 41 f. - Zur PerspektiveDiskussion bei Jan van Eyck zuletzt James Elkins, »On the Arnolfini Portrait and the Lucca-Madonna: Did Jan van Eyck have a perspectival system?«, Art Bulletin 73 (1991), S. 53-62, dort ältere Literatur. - Zur Perspektive bei Petrus Christus vgl. Sander (Anm. 16). S. $168-170$. 


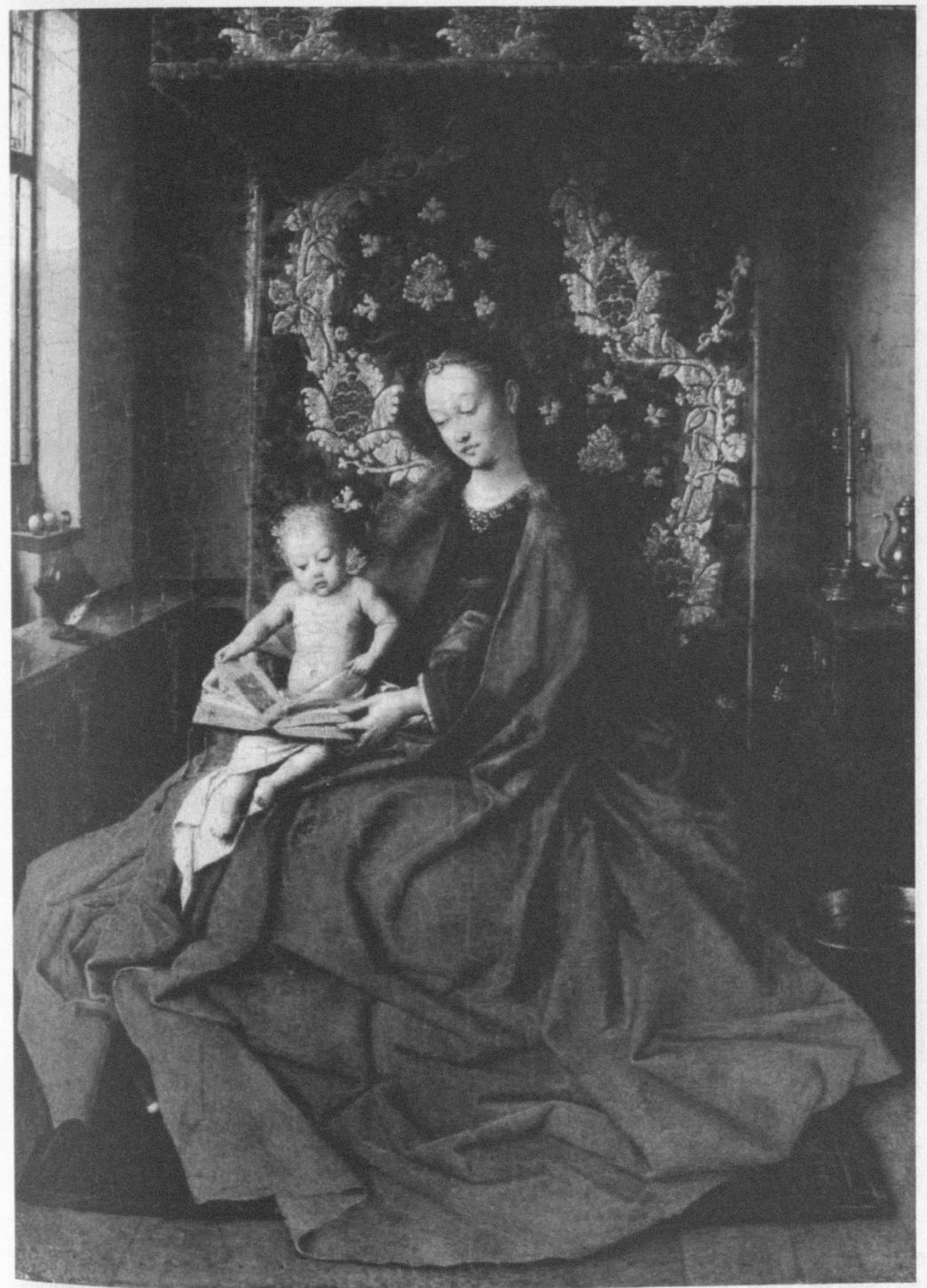

Abb. IV: Nachfolge Jan van Eyck, Ince-Hall-Madonna,

Melbourne, National Gallery of Victoria, Felton Bequest 1922 (Foto: Melbourne, National Gallery). 
Christus den Betrachter [...] auf Distanz «. ${ }^{44}$ Der versteckte ikonographische Symbolismus, der auch in den veristisch wiedergegebenen Gegenständen der Lucca-Madonna zu lesen ist, wird hier in einer solchen Dichte vorgetragen, daB er paradoxerweise eher eine weitere Distanzierung vom Dargestellten bewirkt. Der Thron mit seinen kostbaren, durchsichtigen Säulen, den Reliefs mit den Szenen aus dem Alten Testament, ${ }^{55}$ vor allem aber die aufgezogenen Vorhänge sollen dem Betrachter die Distanz bewußt machen: Ihm wird - so Barbara Lane entsprechend dem Moment der Messe, in dem während der Transsubstantiation der Vorhang den Altar verbarg, der Blick auf das Wunder der Inkarnation eröffnet. ${ }^{56}$ Das Bild wird zur Bühne, auf der das heilige Ereignis so zelebriert wird, $\mathrm{da} B$ es den Zuschauer auf die theologischen und liturgischen Bezüge verweist.

In Jan van Eycks Konzeption allerdings sind, wie schon erwähnt, ebenfalls Elemente der Verfremdung eingebaut, die jedoch allein innerbildliche Wirkung haben. Der Betrachter wird - vergleichen wir etwa die Ince-Hall-Madonna mit der Lucca-Madonna - erst im Bildraum darauf aufmerksam, daß die ihm scheinbar zum Greifen nahe heilige Gruppe nur in einer eigenartigen Kompaktheit und unlebendigen Starrheit sichtbar wird. Die rigide Strenge und Geschlossenheit der Gruppe ermöglichen eine Monumentalität der Maria, die Harbison wohl zu Recht in Zusammenhang mit der zeitgenössischen Diskussion über die immaculata conceptio bringt ${ }^{57}$ und die sie unberührbar erscheinen läßt. Sie wird als Bild im Bild erkannt, scheint doch van Eyck hier mit Assoziationen an eine Statuengruppe zu spielen. ${ }^{58}$ Erst in der intensiven Auseinandersetzung bemerkt der Betrachter, daß die Umgebung der Madonna trotz ihres Verismus mit komplizierten ikonographischen Bezügen besetzt ist und der Raum sich ihm keineswegs als ein einordenbarer erschließt. Ähnlich wie mit seinen Spiegelbildern weist van Eyck den bereits in das Werk eingetretenen Betrachter wieder aus diesem hinaus und macht ihn in versteckten Anspielungen darauf aufmerksam, da $B$ dieses Bild die letzte Wahrheit doch nicht sei.

Van Eycks Distanzierung bleibt infolgedessen eine innerbildliche, gehört doch die Lucca-Madonna zur visuellen Erfahrungswelt des Betrachters; er ist sozusagen stummer Akteur, der die ebenso stumme Figurengruppe in einem scheinbar visionären Akt heraufzubeschwören vermag. ${ }^{59}$ Seine Nachfolger jedoch sind bestrebt, das Bild demonstrativ aus der Welt des Betrachters zu entfernen. In der Ince-Hall-Madonna präsentiert es sich trotz seiner perspektivischen Raumge-

54 Sander (Anm. 16), S. 172. - Ganz anders deutet Upton (Anm. 51), S. 42 diese Darstellung, interpretiert er doch die Richtigkeit der Perspektive als eine Ausweitung des Betrachterraumes, ohne dabei die entsprechende Abgrenzung zu sehen.

55 Die beiden Stammeltern an den Thronwangen verweisen auf Maria und Christus als neue Eva und neuer Adam; in den beiden alttestamentlich gekleideten Gestalten an der Thronrückwand dürften Propheten angesprochen sein, die gemäß der Darstellung mit ihrer Prophezeiung den Vorhang schon geöffnet hatten.

56 Lane (Anm. 52), S. 25.

57 Harbison, Jan van Eyck (Anm. 6), S. 75-85.

58 Ebd., S. 78; vgl. auch Lane (Anm. 52), S. 25.

59 Otto Pächt, Van Eyck. Die Begründer der altmiederländischen Malerei, hrsg. Maria Schmidt-Dengler, München 1989, S. 23 f. 


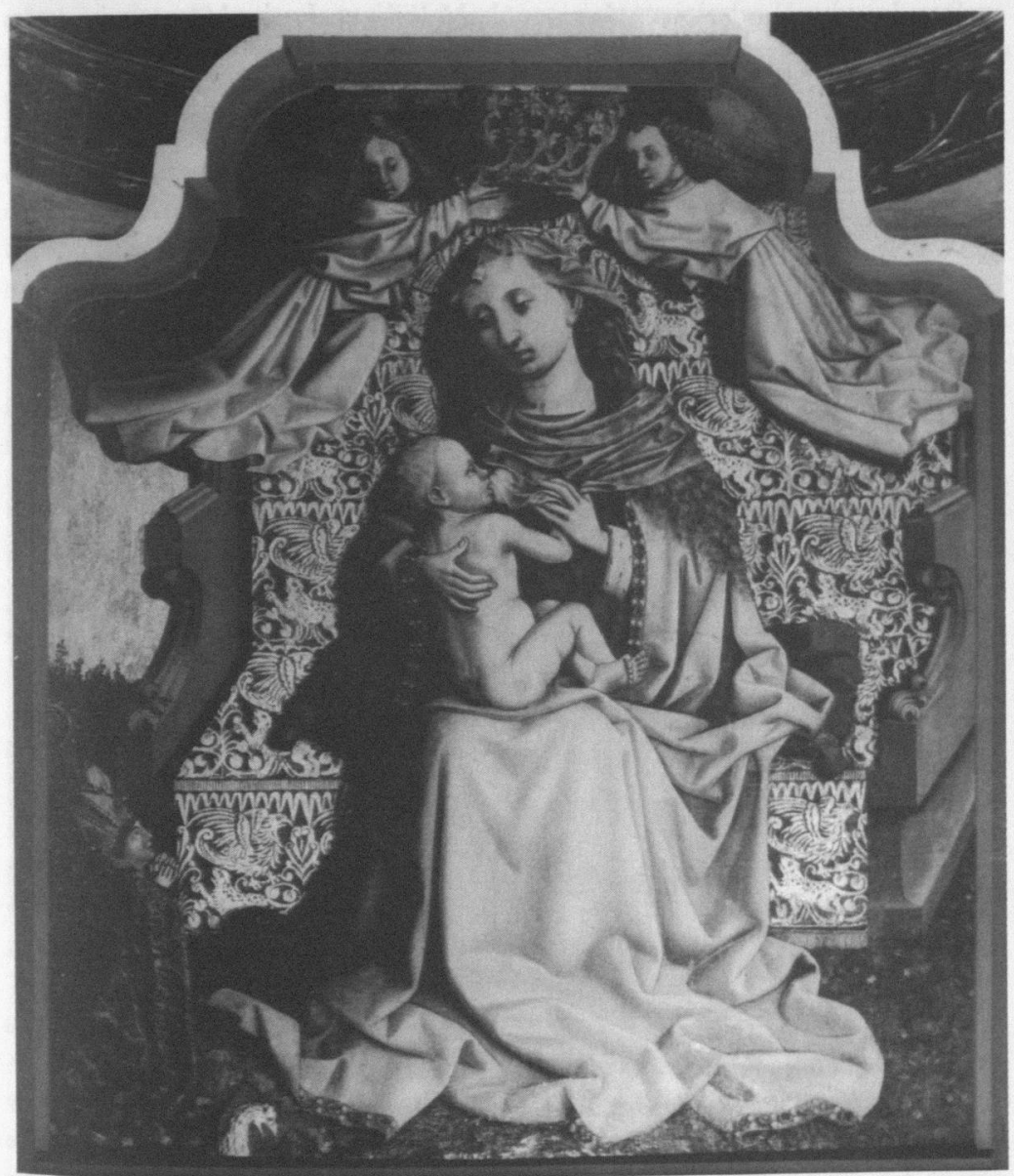

Abb. V: Regensburg(?), Madonna des Abtes Wolfhard Strauss,

Regensburg, St. Emmeram

(Foto: Regensburg, Denkmalamt).

staltung als eine eigene Realität. Bezeichnenderweise werden der Teppich wie auch alle übrigen Objekte deutlich abgesetzt vom Bilderrahmen und in einem ihnen zugehörigen Bereich so situiert, daß der Betrachter nicht eintreten kann, sondern ihm lediglich die Anschauung gewährt wird. Mobiliar und Teppich, vor allem aber der Thron, sind Requisiten geworden, die im Gegensatz zu den Objekten in Jan van Eycks Tafeln nicht dazu dienen, einen Raum - und damit ein Erzählkontinuum - zu schaffen. Unklar bleibt denn auch die räumliche >Ortung ‘ der Madonna mit dem Kind. Sitzt sie nun auf dem Teppich oder auf einem unsichtbaren Stuhl oder Thron? Wo der weit sich ausbreitende knittrige Faltenmantel oder der Frauenkörper sich befindet, wie die Übergröße der Maria sich im Raum zu bewähren hätte, dies alles wird uns verborgen. Nicht zuletzt der breit in die Fläche 


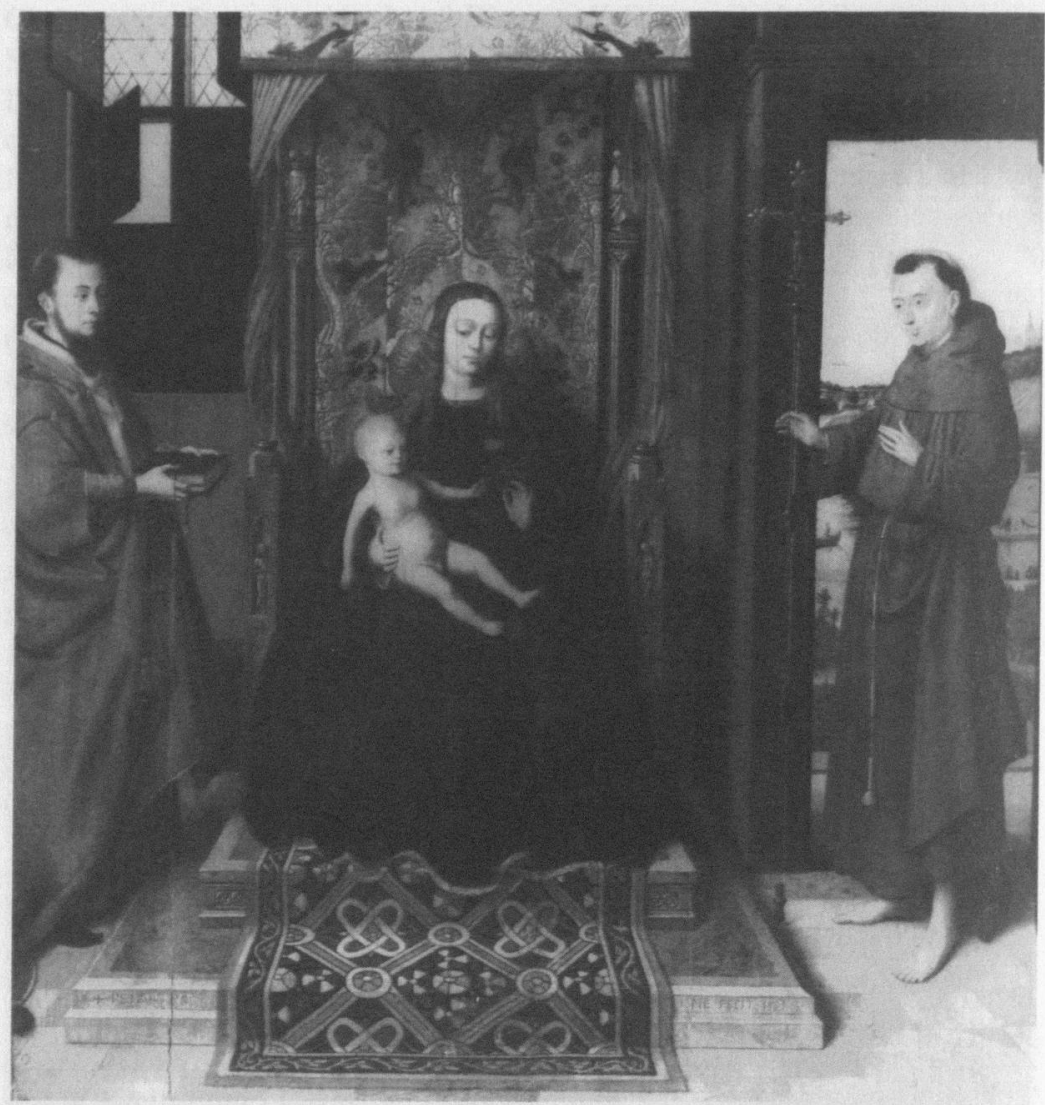

Abb. VI: Petrus Christus, Madonna mit Hieronymus und Franziskus, Städelsches Kunstinstitut Frankfurt/M., Inv. Nr. 920

(Foto: Frankfurt, Ursula Edelmann).

sich ausbreitende, üppige Samtmantel schafft eine Abgrenzung der Madonna gegenüber dem Betrachter, die - wie auch der attributive Raum - bewußt machen, daß die Realität dieses gemalten Raums keine Fortsetzung des Betrachterraums sein will. Er schaut diesen an und tritt nicht hinein. Zum Bühnenraum schließlich wird der Raum in der Darstellung von Petrus Christus, der hier ein tableau vivant präsentiert.

\section{Das Bild spricht zum Betrachter}

In der neu geschaffenen innerbildlichen Welt der Nachfolgewerke von Jan van Eycks Lucca-Madonna hat sich auch das Verhältnis der Figuren zum Betrachter gewandelt. Das ehemals >stille` Bild hat wiederum einen Ansprachecha- 
rakter erhalten. ${ }^{60}$ In der Ince-Hall-Madonna richtet sich das Kind nach außen. Sein Blättern im Buch mahnt den Betrachter, gleiches zu tun. Das Buch und die demonstrativ präsentierte Windel, auf welcher das Kind sitzt, werden hier als Heilswahrzeichen geradezu vorgezeigt. Gerade die Windel, die sowohl die Geburt als auch den Tod, vor allem das Korporale der Messe impliziert, demonstriert dem Betrachter, daß mit Hilfe solcher Zeichen die Wahrheiten von Inkarnation und Erlösung einzig in der Messe übermittelt werden können.

Ähnliche Verweise benutzt auch Petrus Christus, der zwar die Gruppe im Vergleich zu Jan van Eyck scheinbar öffnet, aber zu einer innerbildlichen Kommunikation zwischen Kind und Heiligen gestaltet. Nicht dem Betrachter wird dieses Gespräch zuteil, sondern die heiligen Figuren sehen stellvertretend für ihn auf das visionäre Bild. Madonna wie Kind werden entsprechend der InceHall-Madonna präsentiert. Das Vorzeigen von Buch, Rosenkranz und des im Licht aufblitzenden Stabes des Vortragekreuzes gemahnen klar an den >richtigen $<$ Kontext, der einzig in der Liturgie liegen kann. Nur dort - und darauf verweist auch die Ince-Hall-Madonna - kann auf jene letztgültigen Wahrheiten geschaut werden.

In der Strauss-Madonna scheint das Heilige als eine Aufführung ins Bild übersetzt worden zu sein. Die Staffelung der Darstellung läßt den Stifter zu Füßen der Madonna zum Regisseur werden, der zwischen der Madonnenvision und dem Betrachter vermittelt und diesem als Anleitung und Vorbild dient. In Nachahmung seiner im Bild vorgeführten, verehrenden Haltung hat sich der Betrachter dem in die himmlische Sphäre entrückten Madonnenbild zu nähern.

Gemeinsam war den Nachfolgewerken erstens die Wiederherstellung einer eigenen ikonischen Realität, in der das Heilige aufgeführt wird; zweitens die Ansprache an den Betrachter, indem eine der agierenden Personen sich an ihn wendet oder als Stellvertreter eine gewisse Übermittlung übernimmt; drittens eine attributive Demonstration der einzelnen Inhalte - Buch, Windel, Vortragekreuz, Thron, Brokatvorhang usw. Jeweils unterschiedlich wurde die vierte Veränderung behandelt, nämlich der Einbezug von Handlungsmomenten. Während in der Lucca-Madonna die Handlung im Eintreten des Betrachters besteht, wird in allen Nachfolgewerken diese wiederum im Bild selbst dargestellt. In der Version von Petrus Christus allerdings scheint trotz der hinzugefügten Personen die Handlung im Sinne eines lebenden Bildes eingefroren worden zu sein. In den anderen beiden Varianten jedoch intensivieren das agierende Kind,

60) Zum früheren Wandel vom stillen zum sprechenden Bild vgl. Belting (Anm. 5). S. 20 f.; in einem anderen Kontext vgl. Richard C. Trexler, »Das sprechende Bildnis. Versuch einer Typologie im Spiegel spanischer Quellen des 16. Jahrhunderts «, in: Klaus Schreiner (Hrsg.), Laienfrömmigkeit im späten Mittelalter, Schriften des Historischen Kollegs, Kolloquien 20, München 1992, S. 283-308; obwohl es sich hier um die Berichte der Eroberer über zu den Ureinwohnern sprechende Bilder handelt, läßt sich gerade an solchen Projektionen die auch im 16. Jahrhundert noch vorhandene. besondere Wirksamkeit der bildlichen Ansprache und auch des Dialogs mit dem Bild erkennen. 
die rauschenden Engel und der Stifter die Erzählung in dramatisierender Weise.

Gehen wir zu den eingangs geäußerten Annahmen zurück, so haben sich die Nachfolgewerke der Lucca-Madonna als eine Abkehr von der objektiven, das heißt perspektivisch richtigen, mimetischen Wiedergabe der geschauten Welt erkennen lassen. Damit verbunden ist eine andere Funktion des Bildes. Nicht mit dem Bild soll sich der Betrachter identifizieren, sondern dieses leitet ihn an, in eine außerikonische, höhere Wirklichkeit aufzusteigen. Während in der LuccaMadonna der Betrachter auf einer ihm und dem Bild gemeinsamen Bühne steht, infolgedessen das >Andachtsstück ` ohne seine Anwesenheit nicht vollständig ist, wird in den späteren Bildern der Gegenstand demonstriert und damit wiederum mit einer vom Betrachter unabhängigen Identität versehen. Das Bild bezeichnet eine eigene Wirklichkeit, die den Betrachter in wachsender Eindringlichkeit dazu anhält, seinen Blick auf die übergeordnete Wahrheit zu richten.

Diese Konzepte greifen zwar auf ältere Bildtraditionen zurück, werden allerdings - sind sie doch mit der ganz anderen Bilderfahrung, etwa der >Ars Nova des Jan van Eyck ${ }^{61}$, konfrontiert - in ihren Bezügen auf die übergeordneten Wahrheiten, vor allem der Heiligen Schrift, wie auch in ihren Anweisungen an den Betrachter zur nachagierenden Teilnahme immer intensiver. Diese Dringlichkeit des Appells, sich mit Hilfe des Bildes dem >Eigentlichen` zu nähern, läßt die Maler zu wiederum dem Schauspiel strukturell verwandten Lösungen greifen, beispielsweise zur Bühnenpräsentation und zu Teilrealismen, zur Dramatisierung der Handlung oder Kumulierung von unterschiedlichen Erzählbühnen. ${ }^{6}$

Die erneute Betonung des Bildes als fiktional, die in den hier besprochenen Beispielen die Distanz zum Betrachter mit einer zugleich intensiveren Ansprache kombinieren, kann recht unterschiedliche Formen finden. Keinesfalls kann sie als einlinige Entwicklung etwa im Sinne einer zunehmenden Theatralisierung verstanden werden. Neben höchst dramatischen Wiedergaben eines erschütternden Passionsrealismus werden gerade gegenteilige Formen der Ansprache gesucht. Im Erfurter Regler Altar ${ }^{63}$ beispielsweise wird dem Betrachter in eindrucksvoller Dramatik das Passionsgeschehen wie eines der tatsächlich üblichen Schauspiele im und auf dem Lettner ${ }^{64}$ dargeboten. Der Colmarer Altar des Caspar Isenmann ${ }^{65}$ dagegen, eines Malers, über dessen Beteiligung an Fron-

61 Zur Übertragbarkeit des aus der Musikgeschichte stammenden Begriffes der 'Ars Nova auf die erste Generation der Niederländer vgl. Pochat (Anm. 26), S. $216 \mathrm{f}$.

62 Zur Simultanbühne etwa bei Memling vgl. Pochat (Anm. 1), S. 46 ff.

63 Abb. vgl. Heinrich Theodor Musper, Gotische Malerei nördlich der Alpen, Köln 1961. Abb. 160-162.

$64 \mathrm{Zu}$ den Aufführungen auf und im Lettner vgl. Pochat (Anm. 1), S. 86 ff. Auch hier dient die Fiktion eines Flügelaltars als gemalter Lettner der Verfremdung. Von debattierenden Propheten, Engeln und den Gestalten des Passionsgeschehens bevölkert, ermöglicht der Lettner ein typologisches Programm.

65 Abb. vgl. Sylvie Lecoq-Ramod u. Pantixika Béguerie, Le musée d' Unterlinden de Colmar: Musées et monuments de France, o. O., o. J., S. 44. 
leichnamsspielen wir informiert sind, ${ }^{66}$ verzichtet auf eine dramatisierende Darstellung und reiht Bild an Bild wie einzelne Kulissenversatzstuicke nebeneinander. Das Demonstrieren der Glaubensinhalte, das Vorweisen der individualisierten Trauer der einzelnen Akteure wird hier vorgeführt. ${ }^{67}$ Tendenziell nehmen allerdings die Deutlichkeit der Verweise und oft auch die Drastik der Erzählung zu.68 Da die Aufgaben und wohl auch das Publikum der einzelnen Medien - ja sogar der einzelnen Tafelbilder - oft verschiedenartig und vielschichtig sind, müssen infolgedessen auch die Arten der Ansprache wechseln.

Das hier untersuchte Phänomen einer neuen Fiktionalität des Bildes, die bei den frühen Niederländern mit dem Spiel des über sich selbst hinausweisenden Realismus gesucht wird, während die nächsten Generationen zur innerikonischen Realität zurückkehren, kann nicht allein theologisch erklärt werden. Ebensowenig allerdings vermag die Annahme eines Wandels der Bedürfnisse diesen Vorgängen gerecht zu werden. ${ }^{69} \mathrm{Zwar}$ müssen die Maler die einmal gefundene mimetische Seherfahrung mit einem gewissen Angebot an den Betrachter beantworten, aber womit sollte dann gerade das konservative Element dieser Prozesse

66 Vgl. dazu Friederike Blasius, Bildprogramm und Realität. Untersuchungen zur oberrheinischen Malerei um die Mitte des 15. Jahrhunderts am Beispiel der,Karlsruher Passion<, Frankfurt/M., Bern u. New York 1986, S. 36.

67 Besonders deutlich wird diese Funktion der Bildakteure als Träger individueller Trauerund Mitleidensbotschaften am Beispiel der Grablegung. Hier wird das Bildthema, das sich wohl auf die Grablegung des sogenannten Seilern-Triptychons, London, CourtauldInstitute, bezieht (Abb. vgl. Pächt (Anm. 59), Taf. 2), so abgewandelt, daß jede einzelne Gestalt sich in ihrer jeweils isolierten Trauer nach außen zum Betrachter richtet. Eine ähnliche Hinwendung ist beispielsweise auch beim Meister des Marienlebens im sogenannten De-Monte-Triptychon zu beobachten (Abb. in Hans Martin Schmidt, Der Meister des Marienlebens. Studien zur spätgotischen Malerei in Köln, Beiträge zu den Bau- und Kunstdenkmälern im Rheinland 22, Düsseldorf 1978, Abb. 29 u. 30). - Zu den Einflüssen vgl. Barbara Jakobi, Der Einfluß der niederländischen Tafelmalerei des 15. Jahrhunderts auf die Kunst der benachbarten Rheinlande am Beispiel der Verkündigungsdarstellung in Köln, am Niederrhein und in Westfalen (1440-1490), Kölner Schriften zu Geschichte und Kultur, Köln 1987, S. 123 ff.

68 Eine ähnliche Reihe wie diejenige der Lucca-Madonna ließe sich auch am Beispiel der Trinitätsdarstellung des Robert Campin (Frankfurt, Städel) verfolgen. Die Fiktion des Andachtsbildes im Stein (Sander (Anm. 16), Taf. 7) wird etwa in der St. Petersburger Version zu einem Blick auf die srealen. Wunden Christi, die in einem entschleierten Baldachin dem Betrachter dargeboten werden. Die Situierung des Themas in einem Hoheitsbezirk - in einer späteren Version Colin de Cotters im Himmel von Engeln mit den Leidenswerkzeugen umgeben und schließlich kombiniert mit der Fürbitte von Maria und Johannes - sind ähnliche Stationen der zunehmenden Entrückung, Theatralisierung und narrativen Verdeutlichung; vgl. Rivière (Anm. 37), Abb. 5 u. 6.

69 Diese Lösung bietet der Aufsatz von Thürlemann an, der mit seiner rein ästhetischen Argumentation zweifellos zu einseitig ist: Felix Thürlemann, „Die Madrider Kreuzabnahme und die Pariser Grabtragung: das malerische und zeichnerische Hauptwerk Robert Campins«, Pantheon 51 (1993), S. 18-45, bes. Abb. 12 u. 14-17. - Eine Kombination ganz verschiedener Beweggründe erwägt Harbison, Jan van Eyck (Anm. 6). S. $198 \mathrm{ff}$., wobei auch Überlegungen zur Multifunktionalität von Bildern angestellt werden. 
erklärt werden? Hierfür scheint die Verwendung der Bilder vor allem eine Rolle gespielt zu haben. Dabei müssen wohl entsprechende Vorgänge in der Frömmigkeitspraxis - so etwa derjenigen der devotio moderna ${ }^{70}$ - wie auch die alten Auseinandersetzungen über die Gefahren des Bildes und seiner notwendigen Unterordnung unter die Heilige Schrift mitbedacht werden.

70 Dazu Rivière (Anm. 37), S. 146, der den Wandel »de l'irréalité du gothique international à la Vision Claire des choses [bei Campin|, pour revenir, avec les artistes de la fin $\mathrm{du}$ siècle, à ce que l'on pourrait appeler une réalité contrôlée « allein mit der devotio moderna zu erklären versucht, was angesichts der verschiedenen angebotenen Lösungen ebenfalls zu einseitig erscheint. 